\title{
Separation of Surface Roughness Profile from Raw Contour based on Empirical Mode Decomposition
}

\author{
Shoubin LIU ${ }^{1, a^{*}}$, Hui ZHANG ${ }^{2, b}$ \\ ${ }^{1}$ Department of Mechanical Engineering and Automation, Harbin Institute of Technology Shenzhen \\ Graduate School, Shenzhen, 518055, China \\ ${ }^{2}$ Department of Mechanical Engineering and Automation, Harbin Institute of Technology Shenzhen \\ Graduate School, Shenzhen, 518055, China \\ aemail: mesbliu@hitsz.edu.cn, bemail:zhanghui369963@163.com
}

Keywords: Surface Roughness Profile; EMD; IMFs; Mean Line

\begin{abstract}
Engineering surfaces are comprised of different wavelength bands commonly referred to as roughness, waviness and form. The Gaussian filter is a common technique to separate surface roughness profile but it has some drawbacks. This paper presents an approach of utilizing Empirical Mode Decomposition (EMD) to separate surface roughness profile from raw contour. The EMD describes a time-signal with a series of Intrinsic Mode Functions (IMFs) and the residue. The residue and IMFs of frequency less than 10 within an evaluation length, are used for reconstructing EMD reference line. To achieve more accurate mean line, an Amplitude Weighting Factor (AWF) $\mu$ is applied to the IMF whose frequency is maximum among the selected IMFs. The EMD reference line is thus modified as EMD mean line. Surface roughness profile can be further separated and roughness parameters can be calculated. The comparison of EMD method and Gaussian filter is also presented.
\end{abstract}

\section{Introduction}

A typical engineering surface consists of roughness, waviness and form [1], which influences the performance of mechanical system [2]. To evaluate various parameters of surface profile, it is necessary to extract surface roughness profile. The separation of roughness profile is usually based on a mean line and filtering methods are commonly adopted to determine the mean line. The filters include 2RC filter, Gaussian filter, Spline filter and Wavelet filter [3].

Although the 2RC filter is still applied in traditional instruments, the filter has been deleted from ISO standard since its inherent defects. Gaussian filter is the most common filter in the assessment of surface morphology, but it suffers from edge distortion effects [4] [5]. The Spline and the Wavelet filters are recent advances in filtering of surface morphology. The Spline filter gets the reference line by a simple closed formula [6]. The Wavelet filter is a multi-resolution decomposition method but the analyzing results to a same engineering surface are different with different wavelet bases [7].

Empirical Mode Decomposition (EMD) is proposed by Huang and can be used to analyze nonlinear and time-varying signal [8]. The EMD is self-adaptive and doesn't need any basis function. A complex signal can be decomposed into a series of Intrinsic Mode Functions (IMFs) and the residue. The decomposition is based on the local characteristic time scale of the signal. In theory, the EMD method can be used for decomposition of any type of time-signal, especially for processing non-stationary and nonlinear signals. So, the method was applied widely as soon as it was put forward.

As shown in Figure 1 (a), a profilometer is used to measure a surface of a workpiece. The measured raw contour is shown in Figure 1(b) where the roughness profile rides the trend profile of raw contour. In this paper, the EMD method is used to decompose the raw contour. The decomposed signals with low frequency are selected to reconstruct the EMD mean line. The roughness profile is thus separated and roughness parameters are calculated. 


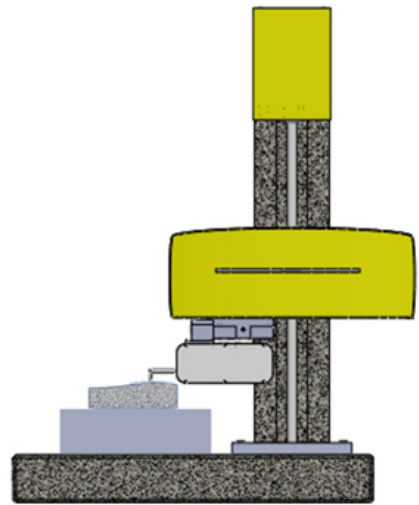

(a)

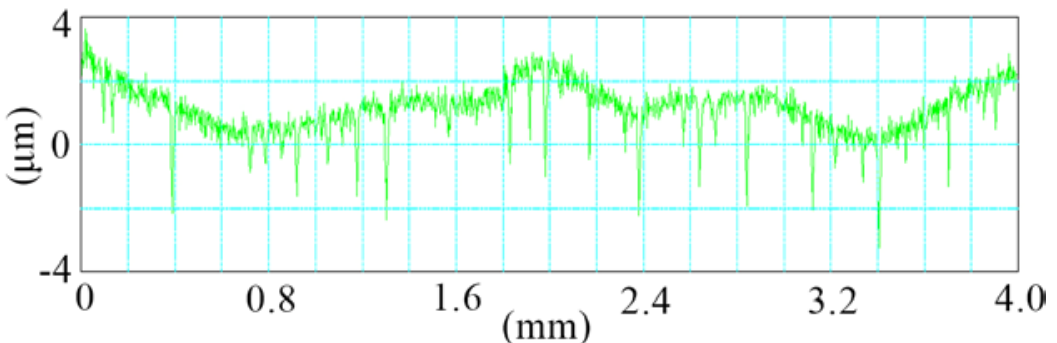

(b)

Fig. 1. A profilometer (a), and the measured raw contour (b)

\section{Empirical Mode Decomposition of Raw Contour}

The EMD considers that a signal is composed of many components called IMFs. The implementation of EMD is a sifting process resulting in IMFs. For a signal $x(t)$, the sifting process is described as follows [8]:

1) Identify all extrema of $x(t)$.

2) Find the maximum envelope $e_{\max }(t)$ of $x(t)$ by passing a natural cubic spline through the maxima. Similarly find the minimum envelope $e_{\min }(t)$ with the minima.

3) Compute the mean, $r(t)=\left(e_{\max }(t)+e_{\min }(t)\right) / 2$.

4) Compute the new sequence $m_{i}(t)=x(t)-r(t)$.

5) Check whether $m_{i}(t)$ is an IMF. If $m_{i}(t)$ is not an IMF, repeat the loop on $m_{i}(t)$. If $m_{i}(t)$ is an IMF then set $c_{i}(t)=m_{i}(t)$.

The process will be terminated until either the residue, $r_{n}$, becomes a monotonic function from which no more IMF can be extracted, or when the IMF, $c_{n}(t)$, or the residue, $r_{n}$, becomes so small that it is less than the predetermined value [8]. Once one of these criteria is satisfied, $n$-empirical modes of the signal are extracted. Then we obtain an expression of $x(t)$ as

$$
x(t)=\sum_{i=1}^{n} c_{i}(t)+r_{n}(t)
$$

Where $r_{n}(t)$ is the residue but not an IMF, $c_{i}(t)$ is an IMF, a special wave with varied amplitude. Based on the sifting process of EMD, the raw contour shown in Figure 1(b) can be decomposed into a series of IMFs and the residue. Several last IMFs and the residue are shown in Figure 2.

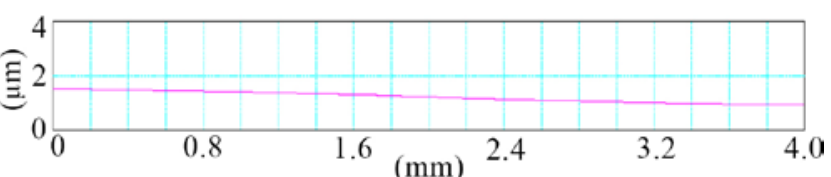

(a) Residue

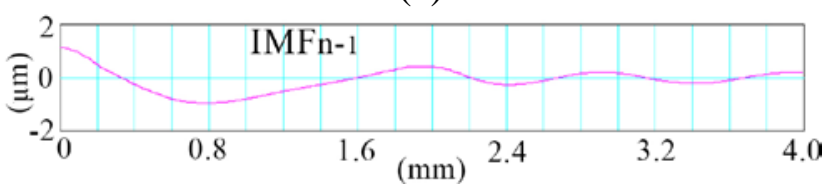

(c) $\mathrm{IMF}_{\mathrm{n}-1}$

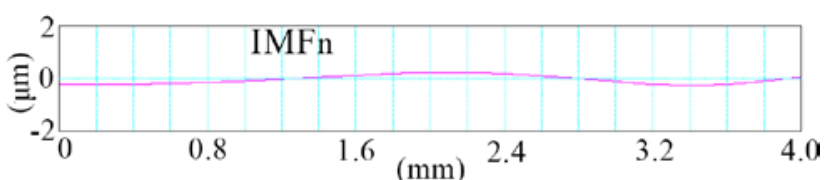

(b) $\mathrm{IMF}_{\mathrm{n}}$

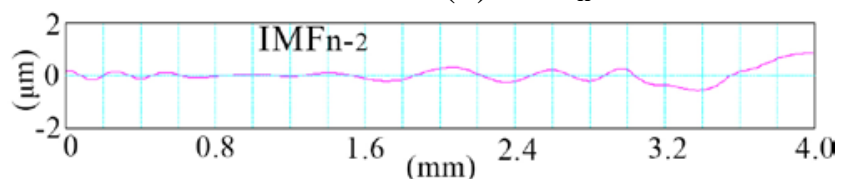

(d) $\mathrm{IMF}_{\mathrm{n}-2}$

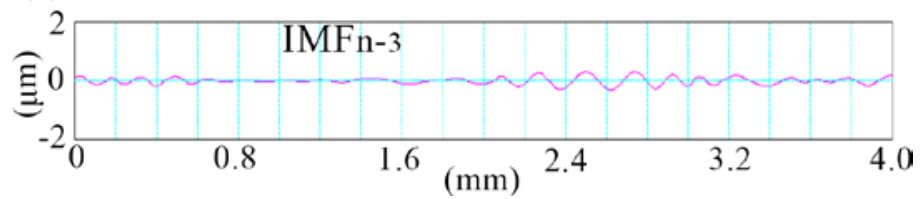

(e) $\mathrm{IMF}_{\mathrm{n}-3}$

Fig. 2. Several IMFs of low frequency and the residue 


\section{Generation of EMD Reference Line}

Since the low frequency represents the trend factor of raw contour, and the frequency of an IMF will become lower with the increasing of its index number, we use several IMFs of low frequency and the residue, $r_{n}(t)$ to reconstruct the EMD reference line of surface profile. As shown in Figure 3, the black curve, $\mathrm{L}_{1}$ is formed by $r_{n}(t), \mathrm{IMF}_{\mathrm{n}}$ and $\mathrm{IMF}_{\mathrm{n}-1}$. The purple curve, $\mathrm{L}_{2}$ is formed by $r_{n}(t)$, $\mathrm{IMF}_{\mathrm{n}}, \mathrm{IMF}_{\mathrm{n}-1}$ and IMF $\mathrm{IM}_{\mathrm{n}-2}$. The blue curve, $\mathrm{L}_{3}$ is formed by $r_{n}(t), \mathrm{IMF}_{\mathrm{n}}, \mathrm{IMF}_{\mathrm{n}-1}, \mathrm{IMF}_{\mathrm{n}-2}$ and IMF $\mathrm{I}_{\mathrm{n}-3}$.

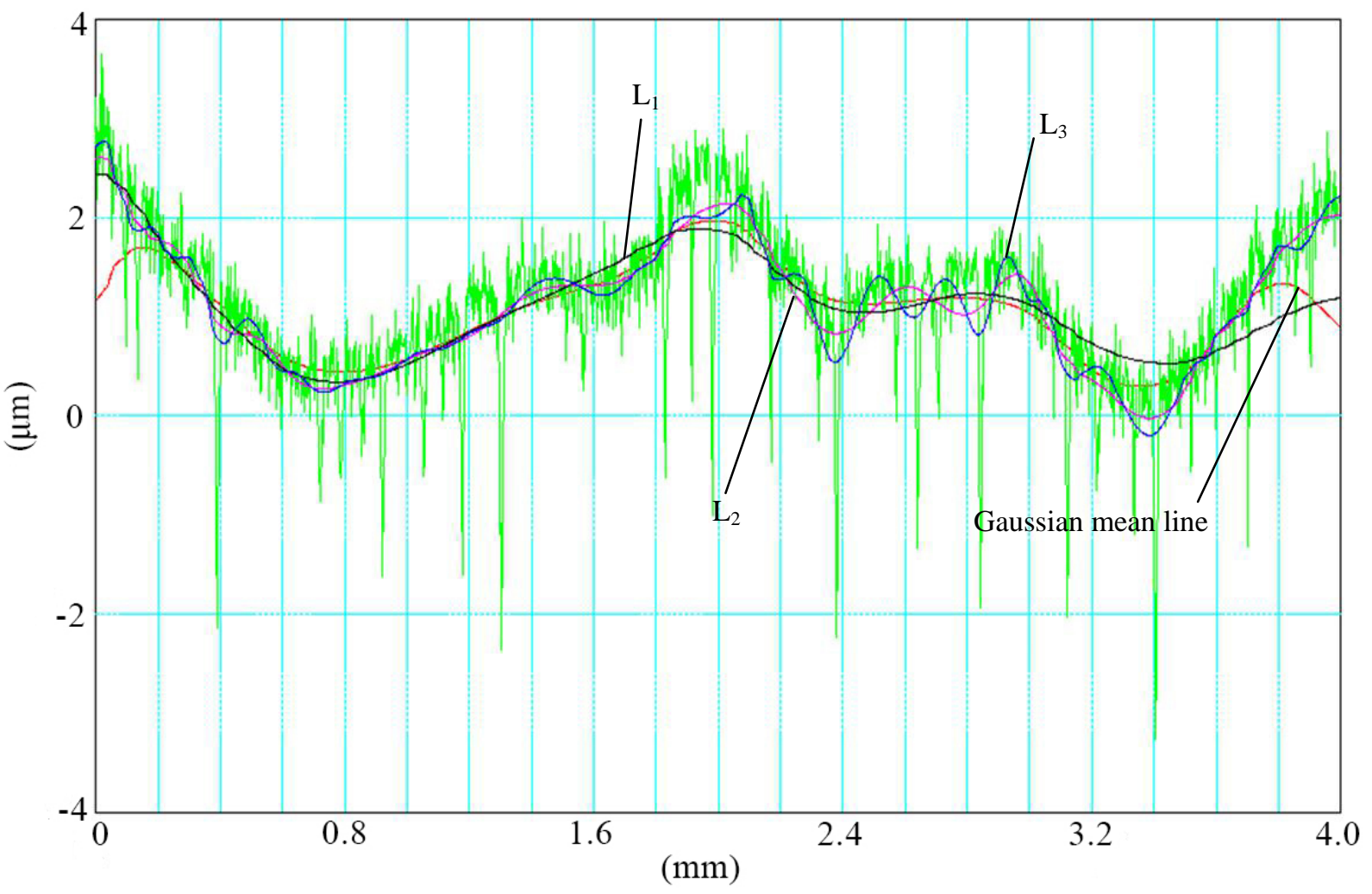

Fig. 3. The comparisons between various reference lines and Gaussian mean line

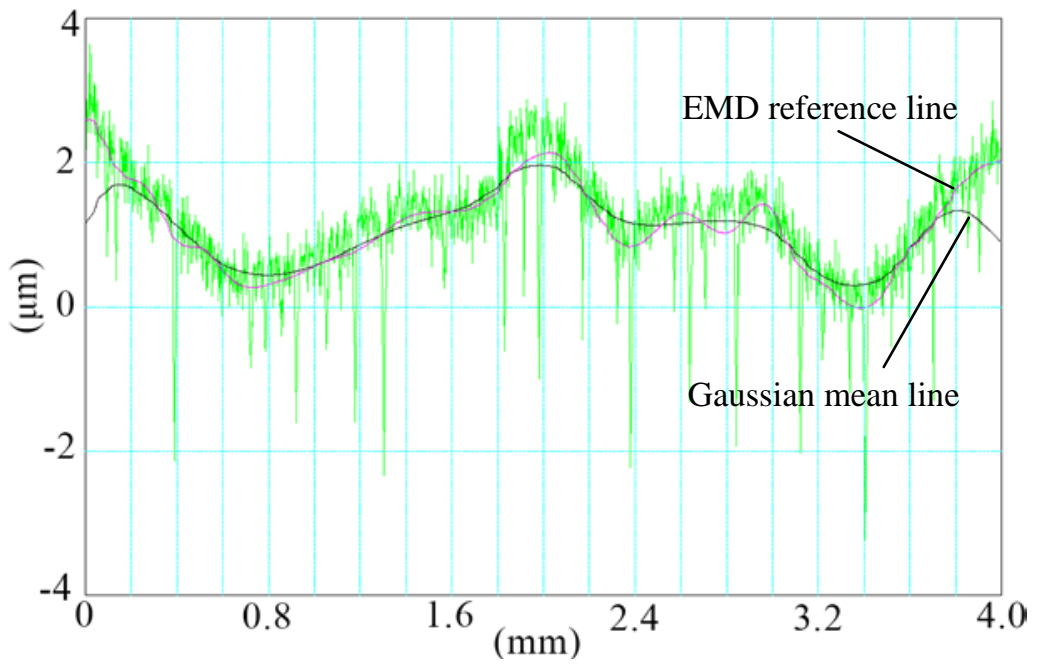

Fig. 4. A comparison of EMD reference line formed by $r_{n}(t), \mathrm{IMF}_{\mathrm{n}}, \mathrm{IMF}_{\mathrm{n}-1}$ and $\mathrm{IMF}_{\mathrm{n}-2}$, and Gaussian mean line

The most widely used filter for surface profile analysis is the Gaussian filter. The weighting function of the Gaussian filter is given by

$$
h(x)=\frac{1}{\alpha \lambda_{c}} e^{-\pi\left(x / \alpha \lambda_{c}\right)^{2}}
$$

Where $\alpha=0.4697, x$ is the position from the origin of the weighting function and $\lambda_{c}$ is the cut-off 
length. In this case, a cut-off length of $\lambda_{c}=0.8 \mathrm{~mm}$ is selected for the measured raw contours.

The convolution of Gaussian weighting function and the raw contour will result in Gaussian mean line as

$$
\omega(x)=y(x) \bullet h(x)=\int_{-\lambda_{c}}^{\lambda_{c}} h\left(x_{t}\right) y\left(x+x_{t}\right) d x_{t}
$$

Where $\omega(x)$ is Gaussian mean line, $y(x)$ is the raw contour, $h(x)$ is the Gaussian weighting function.

As shown in Figure 3, the red one is Gaussian mean line. It is distorted at two end areas due to local weighed average. The blue one, $\mathrm{L}_{3}$ has more fluctuations and the black one, $\mathrm{L}_{1}$ is too smooth at the concave and convex areas. We find that the purple curve, $\mathrm{L}_{2}$ is an appropriate one. It follows the trend of raw contour but has a few fluctuations around Gaussian mean line. We thus define $\mathrm{L}_{2}$ as the EMD reference line, as shown in Figure 4.

According to Figure 3, we conclude that the reference line can be reconstructed by using the residue, $I M F_{n}, I M F_{n-1}$ and $I M F_{n-2}$. The frequency of these IMFs is less than or equal to 8. In addition, the trend frequency of surface roughness profile is usually less than 2 within a cut-off length. It should be less than 10 within an evaluation length supposing that an evaluation length is composed of five cut-off lengths. As a result, we define a principle that the IMFs of frequency less than 10 within an evaluation length are selected to reconstruct the EMD reference line. In general, the frequency of $\mathrm{IMF}_{\mathrm{n}}, \mathrm{IMF}_{\mathrm{n}-1}, \mathrm{IMF}_{\mathrm{n}-2}$, satisfy the principle. Sometimes only $\mathrm{IMF}_{\mathrm{n}}$ and $\mathrm{IMF}_{\mathrm{n}-1}$ satisfy due to the mode mixing phenomenon of EMD.

\section{Amplitude Weighting Factor (AWF)}

To achieve a more accurate mean line, an Amplitude Weighting Factor (AWF) $\mu$ is applied to the IMF whose frequency is maximum among the selected IMFs, i.e. the $\mathrm{IMF}_{\mathrm{n}-2}$ as shown in Figure 2. Then, the mean line is given by

$$
\text { Mean }=r_{n}(t)+c_{n}(t)+c_{n-1}(t)+\mu \bullet c_{n-2}(t)
$$

Where Mean is the mean line, $r_{n}(t)$ is the residue, $c_{n}(t), c_{n-1}(t)$ and $c_{n-2}(t)$ are the IMFs of frequency less than $10, \mu$ is an AWF ranged from 0.0 to 1.0 with a fixed value in the program, $\mu^{\bullet} \mathrm{C}_{n-2}(t)$ is a modified IMF with same frequency.

By formula (4), different $\mu$ will result in different mean line. Based on a mean line, surface roughness profile can be extracted and roughness parameters are calculated. Based on Gaussian mean line via Gaussian filtering, surface roughness profile and parameters are also calculated. The relative error, $E_{r}$ is calculated as

$$
E_{r}=\frac{\left|P_{E M D}-P_{G}\right|}{P_{G}} \times 100 \%
$$

Where $P_{G}$ is the parameter value based on Gaussian mean line, $P_{E M D}$ is the parameter value based on EMD mean line. After Gaussian filtering, roughness parameter $R a$ is $0.3467 \mu \mathrm{m}$. Under different $\mu$, roughness parameter $R a$ and relative errors are calculated based on EMD mean line, as shown in Table 1.

Table 1 Roughness parameter $R a$ calculated based on EMD mean line under different $\mu$

\begin{tabular}{cccccccccccc}
\hline$\mu$ & 0.0 & 0.1 & 0.2 & 0.3 & 0.4 & 0.5 & 0.6 & 0.7 & 0.8 & 0.9 & 1.0 \\
\hline $\begin{array}{c}R a \\
(\mu \mathrm{m})\end{array}$ & 0.3632 & 0.3582 & 0.3537 & 0.3499 & 0.3466 & 0.3440 & 0.3419 & 0.3408 & 0.3403 & 0.3405 & 0.3408 \\
$E_{r}$ & $4.76 \%$ & $3.32 \%$ & $2.02 \%$ & $0.92 \%$ & $0.03 \%$ & $0.78 \%$ & $1.38 \%$ & $1.70 \%$ & $1.85 \%$ & $1.79 \%$ & $1.70 \%$ \\
\hline
\end{tabular}

According to Table 1 , errors of $R a$ are less than $1 \%$ when $\mu$ ranges from 0.3 to 0.5 . We thus give a recommended $\mu$ ranged from 0.3 to 0.5 . In Table 1 , we can find that the error of $R a$ is minimum when $\mu$ equals to 0.4 . Then we reconstruct a more accurate reference line, referred as EMD mean line, as shown in Figure 5(a). The separated surface roughness profile based on EMD mean line is shown in Figure 5(b). 


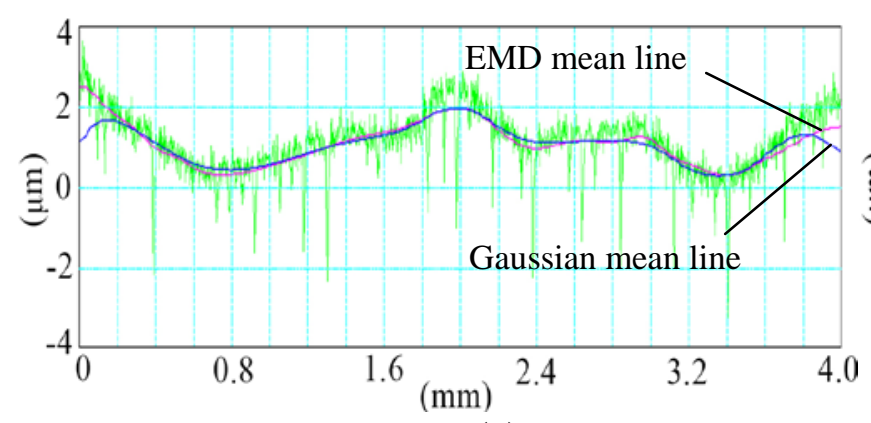

(a)

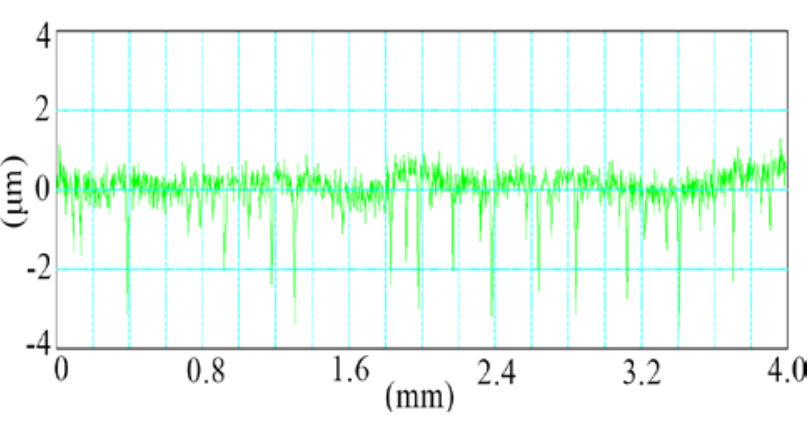

(b)

Fig. 5. Gaussian mean line and EMD mean line with $\mu=0.4$ (a), and roughness profile (b)

\section{Experiment}

Another measured raw contour is shown in Figure 6 . The evaluation length of $l_{n}=4 \mathrm{~mm}$ and the cut-off length of $\lambda_{c}=0.8 \mathrm{~mm}$ are adopted in Gaussian filtering. We first extract the IMFs and the residue of the raw contour. The residue and the last three IMFs are presented in Figure 7.

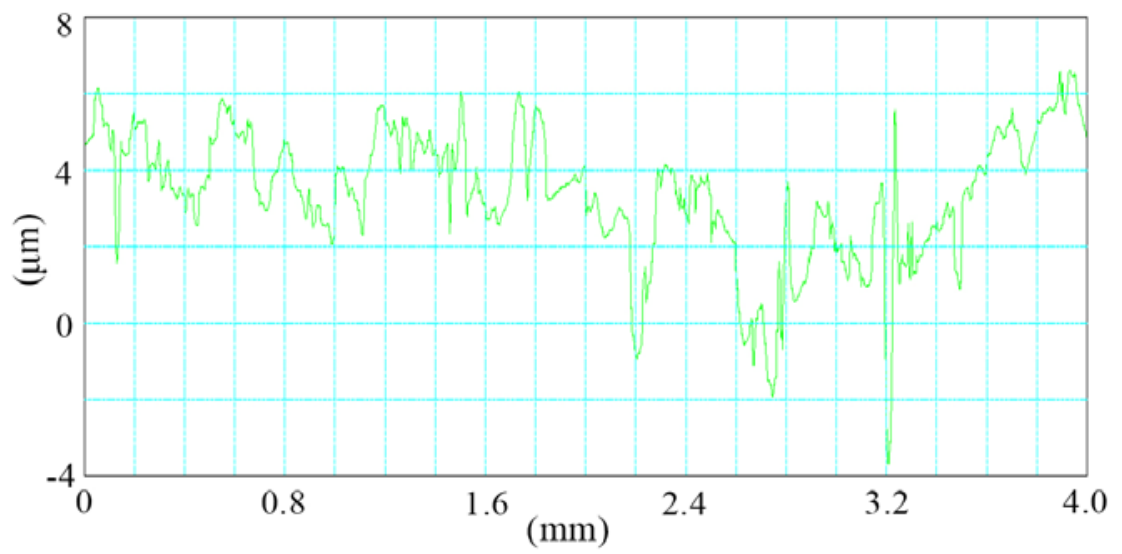

Fig. 6. A raw contour of steel surface

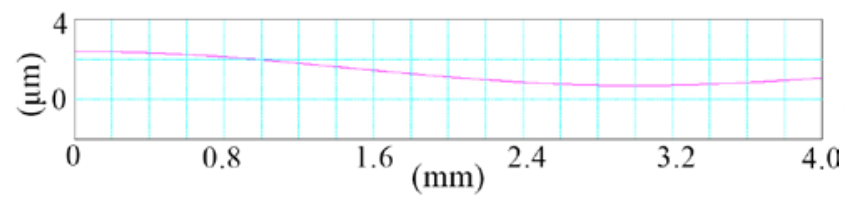

(a) Residue

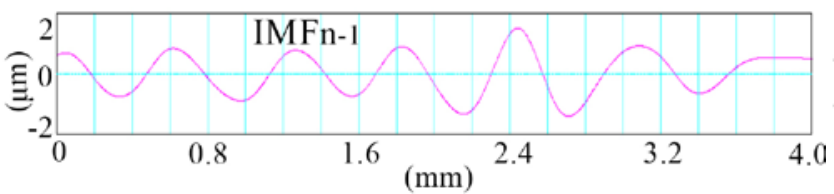

(c) $\mathrm{IMF}_{\mathrm{n}-1}$

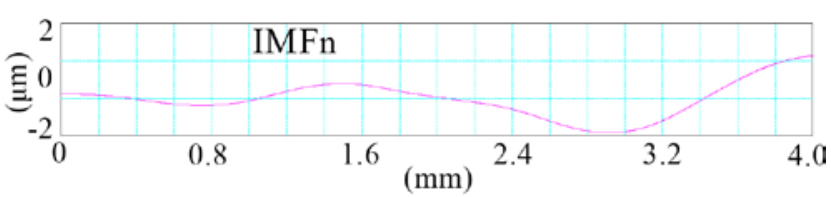

(b) $\mathrm{IMF}_{\mathrm{n}}$

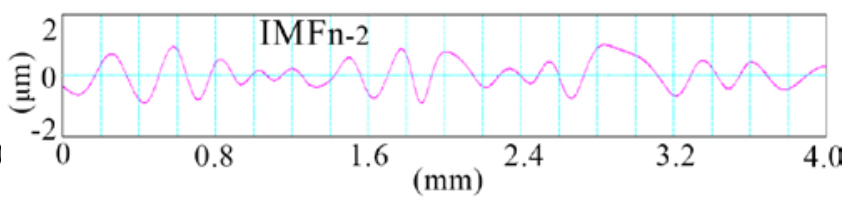

(d) $\mathrm{IMF}_{\mathrm{n}-2}$

Fig. 7. The residue and the last three IMFs

According to the principle that the IMFs of frequency less than 10 within an evaluation length will be selected to reconstruct the EMD reference line, only $\mathrm{IMF}_{\mathrm{n}}$ and $\mathrm{IMF}_{\mathrm{n}-1}$ are satisfied. $\mathrm{IMF}_{\mathrm{n}-1}$ is the one whose frequency is maximum. To obtain a more accurate EMD mean line, $\mu$ is applied to $\mathrm{IMF}_{\mathrm{n}-1}$. Then, the EMD mean line is reconstructed by

$$
\text { Mean }=r_{n}(t)+c_{n}(t)+\mu \bullet c_{n-1}(t)
$$

Where Mean is the EMD mean line, $r_{n}(t)$ is the residue, $c_{n}(t)$ and $c_{n-1}(t)$ are the IMFs. Based on the recommended value of $\mu$, we choose $\mu=0.3$.

According to Figure 8(a), it is obvious that EMD mean line correlates well with Gaussian mean line in most locations. And EMD mean line has less edge effect and can well follow the concave or convex areas. Based on EMD mean line, the surface roughness profile is separated, as shown in Figure 8 (b). Its roughness parameter $R a$ is $0.8335 \mu \mathrm{m}$. Based on Gaussian mean line, roughness parameter $R a$ is $0.8285 \mu \mathrm{m}$. The relative error $E_{r}$ is $0.60 \%$. 


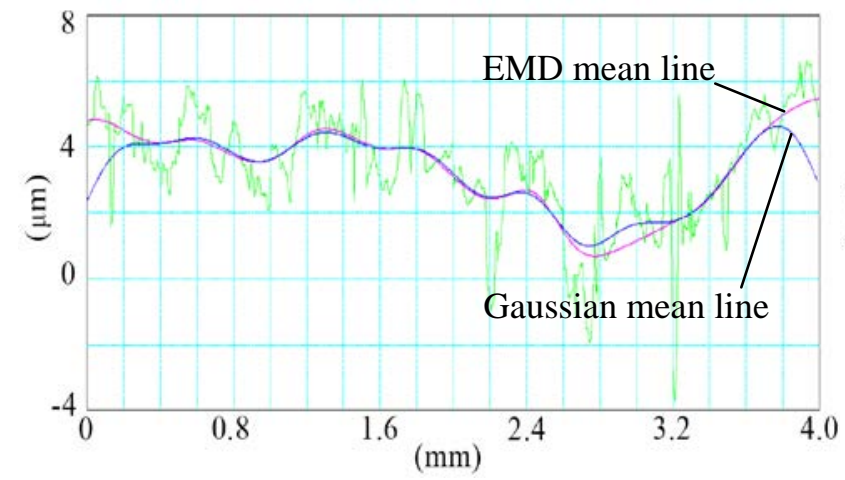

(a) Gaussian mean line and EMD mean line with $\mu=0.3$

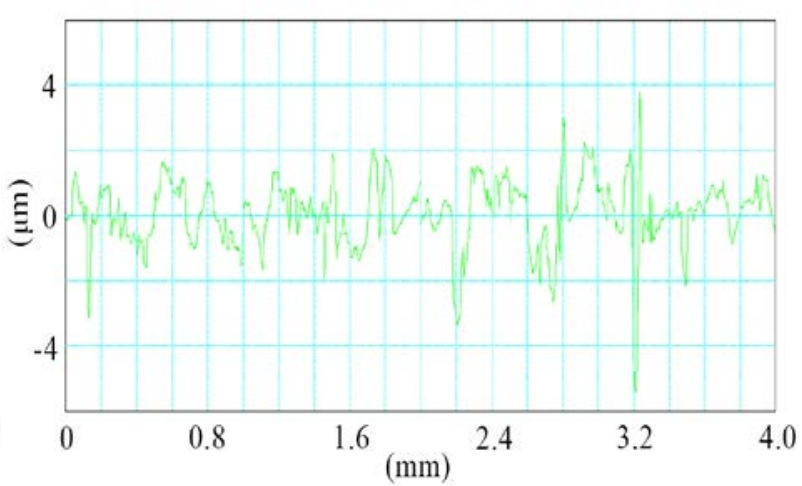

(b) Surface roughness profile

Fig. 8. The EMD mean lines and the extracted roughness profile

\section{Conclusion}

An EMD method is proposed to separate surface roughness profile from raw contour. The residue, several IMFs with frequency less than 10, and an amplitude weighting factor $\mu$ are used to reconstruct the EMD mean line. The EMD mean line follows the trend of raw contour well, without running-in and running-out in the marginal areas of the profile. It correlates well with Gaussian mean line in most locations but is superior in the marginal areas of the profile. In our experiment, EMD mean line will be more accurate when the value of $\mu$ is in the range of 0.3 to 0.5 . Therefore the EMD mean line is recommended by the present authors for separating surface roughness profile.

\section{Acknowledgement}

This research was sponsored by the National Natural Science Foundation of China under grant No. 60873141 and the Shenzhen Basic Research Project grant No. JCYJ20140417172417154.

\section{References}

[1] Boryczko A. Distribution of roughness and waviness components of turned surface profiles[J]. Metrology \& Measurement Systems, 2010, 17(4):611-620.

[2] Jiang X, Scott P, Whitehouse D, et al. Paradigm shifts in surface metrology. Part I. Historical philosophy[J]. Proceedings of the Royal Society A: Mathematical, 2007, 463(2085):2049-2070.

[3] Raja J, Muralikrishnan B, Fu S. Recent advances in separation of roughness, waviness and form[J]. Precision Engineering, 2002, 26(2):222-235.

[4] ISO 11562. Geometrical product specification (GPS)-surface texture: profile method-metrological characteristics of phase correct filters. Geneva: International Organization for Standardization, 1996.

[5] Yuan Y, Qiang X, Song J, et al. A fast algorithm for determining the Gaussian filtered mean line in surface metrology[J]. Precision Engineering, 2000, 24:62-69.

[6] ISO/DTS 16610-22. Geometrical Product Specifications (GPS)-Filtration-Part 22: Linear profile filters: Spline filters. 2003.

[7] Fu S, Muralikrishnan B, Raja J. Engineering surface analysis with different wavelet bases[J]. Journal of Manufacturing Science and Engineering, 2003, 125(4): 844-852.

[8] Huang N, Shen Z, Long S, Wu M, et al. The empirical mode decomposition and the Hilbert spectrum for nonlinear and non-stationary time series analysis[J]. Proceedings of the Royal Society of London A, 1998, 454: 903-995. 